\title{
Impaired efferocytosis by monocytes in multiple myeloma
}

\author{
YING YU LIANG $^{1,5}$, ILSE SCHWARZINGER ${ }^{2}$, INGRID SIMONITSCH-KLUPP ${ }^{3}$, \\ HERMINE AGIS $^{4}$ and RUDOLF OEHLER ${ }^{1}$
}

\author{
Departments of ${ }^{1}$ Surgery and Comprehensive Cancer Center, ${ }^{2}$ Laboratory Medicine, ${ }^{3}$ Pathology and \\ ${ }^{4}$ Internal Medicine I and Comprehensive Cancer Center, Medical University of Vienna, A-1090 Vienna, Austria
}

Received April 21, 2017; Accepted August 8, 2017

DOI: $10.3892 / \mathrm{ol} .2018 .8620$

\begin{abstract}
Efficient clearance of apoptotic cells by efferocytosis is important for tissue homeostasis. Impaired efferocytosis leads to the accumulation of cell debris, which is regarded as a trigger in chronic inflammation and autoimmune diseases. Patients with hematological neoplastic disorders such as multiple myeloma (MM) exhibit high blood levels of apoptotic microparticles. The present study investigated whether these high levels of apoptotic microparticles are associated with insufficient dead cell clearance. Blood samples were collected from patients with MM immediately prior to and 3, 7 and 10 days after the initial cycle of bortezomib-based therapy. In addition, bone marrow aspirates (BMA) were collected prior to and following therapy. Prior to therapy, a 52\% reduction in efferocytosis by blood monocytes was observed compared with the healthy controls $(\mathrm{P}<0.017)$. This was associated with an elevated number of $7-\mathrm{AAD}^{+}$dead cell remnants in the blood flow as well as in BMA. A portion of the blood samples contained active caspase 3 . The subsequent bortezomib-based therapy had no effect on efferocytosis, although the quantity of dead cell remnants decreased. This reduction was associated with a decline in cluster of differentiation $8(\mathrm{CD} 8)^{+}$and $\mathrm{CD} 4^{+}$ $\mathrm{T}$ cells and an increase in the number of monocytes. However, of 28 distinct soluble immune-modulating molecules (i.e. chemokines, cytokines and soluble co-stimulators) only C-C motif chemokine ligand 2 (CCL2), CCL24 and sCD27 were affected by bortezomib-based therapy. The levels of all other molecules remained unchanged or were below the detection threshold in all samples. The present study results revealed
\end{abstract}

Correspondence to: Dr Rudolf Oehler, Department of Surgery and Comprehensive Cancer Center, Medical University of Vienna, AKH, Waehringer Guertel 18-20, A-1090 Vienna, Austria E-mail: rudolf.oehler@meduniwien.ac.at

Present address: ${ }^{5}$ Department of Oncology-Pathology, Karolinska University Hospital, Z:00, SE-171 76 Stockholm, Sweden

Abbreviations: MM, multiple myeloma; BMA, bone marrow aspirates; PBMCs, peripheral blood mononuclear cells

Key words: multiple myeloma, apoptosis, efferocytosis, monocytes, dead cell clearance that the presence of dead cell remnants in the blood and bone morrow of patients with MM is associated with impaired efferocytosis by monocytes; however, its contribution to inflammatory events during MM remains unclear.

\section{Introduction}

Under normal conditions, dying cells are immediately eliminated by professional phagocytes in a process termed efferocytosis (from the Latin efferre, meaning 'to bury'). The molecular machinery of apoptotic cell recognition and uptake by phagocytes has previously been reviewed (1-3). Successful efferocytosis suppresses the activation of nuclear factor $\kappa$-light-chain-enhancer of activated B cells within the phagocyte, inhibits the secretion of tumor necrosis factor (TNF) and promotes the release of interleukin-4 (IL-4), IL-10 and transforming growth factor- $\beta$ (4-6). These mediators are known to induce an alternative-activated macrophage phenotype (7). This cell polarization suppresses inflammation and promotes tissue remodeling and angiogenesis, thereby contributing to the quiescent clearance of apoptotic cells. However, if the clearance of apoptotic cells fails, these cells may enter the late apoptotic/secondary necrotic stage (8). This condition is characterized by a disintegrated cell membrane, leading to the leakage of inflammatory intracellular danger-associated molecular patterns (DAMPs), such as calreticulin, high-mobility group box 1 (HMGB1) and nucleotides. The accumulation of late apoptotic/secondary necrotic cells and the prolonged release of intracellular components from dying cells contributes to chronic or autoimmune diseases (2). Accordingly, impaired efferocytosis has been observed in patients suffering from systemic lupus erythematosus (SLE) (2), chronic granulomatous disease (9), Sjögren's syndrome (10), chronic obstructive pulmonary disease (COPD) (11) and non-eosinophilic asthma (12). A previous study revealed, using genetically modified mouse models, that the absence of genes for vital elements of the dead cell recognition and clearance machinery, such as the Clq complex and milk fat globule-EGF factor 8 , results in an autoimmune phenotype that resembles SLE (13). This suggests the presence of a causal association between reduced efferocytosis and these diseases.

Multiple myeloma (MM) accounts for $\sim 10 \%$ of malignant hematological disorders (14). MM is characterized by the clonal proliferation of cluster of differentiation $138(\mathrm{CD} 138)^{+}$ 
malignant plasma cells in the bone marrow, aberrant production of immunoglobulins, osteolytic lesions, osteopenia and intractable bone pain (14). MM and various other hematological disorders, including chronic lymphocytic leukemia, non-Hodgkin's lymphoma and acute myeloid leukemia, are characterized by high quantities of extracellular vesicles in the blood (15). The number of circulating extracellular vesicles has been recently proposed to be a novel diagnostic marker for MM (16). Between 45 and $95 \%$ of these vesicles are positive for phosphatidylserine, which is a hallmark of apoptosis (15). This suggests that apoptotic microparticles contribute substantially to the circulating extracellular vesicles. However, the reason why apoptotic microparticles accumulate in MM remains unknown. Patients with MM are treated, dependent on their age, with high-dose induction chemotherapy with or without subsequent autologous hematopoietic stem-cell transplantation. The most common induction chemotherapy regimens are bortezomib-based and can be supplemented with dexamethasone, doxorubicin, cyclophosphamide, lenalidomide or thalidomide $(17,18)$. The efficacy of bortezomib-containing induction regimens, with respect to progression-free and overall survival has been confirmed in a meta-analysis of various phase III trials with transplant-eligible myeloma patients (19). Bortezomib is a proteasome inhibitor that induces caspase activation and apoptotic cell death (20). In addition, it inhibits DNA repair and restores the sensitivity of plasma cells to DNA-damaging chemotherapeutic agents. Various in vitro studies have indicated that bortezomib affects also immune cell function. Straube et al (21) demonstrated that bortezomib efficiently impairs the in vitro maturation of dendritic cells (DCs) and blocks the release of TNF and IL-12 upon lipopolysaccharide (LPS) stimulation $(20,21)$. In addition, it was revealed that bortezomib selectively depletes monocytes in peripheral blood mononuclear cell (PBMC) cultures, decreases the survival of purified monocytes and induces apoptosis in monocyte-derived DCs (22). These in vitro data indicated that bortezomib-based regimens have, in addition to their pro-apoptotic effect on malignant plasma cells, an inhibitory influence on monocytes and macrophages; these cells are particularly important for the efficient clearance of dead tumor cells. In a previous study it was demonstrated in vitro that monocytes eliminate late apoptotic/secondary necrotic cells considerably faster than the early apoptotic cells (23). In addition, monocytes secrete distinct cytokines when exposed to late apoptotic/secondary necrotic cell remnants compared with early apoptotic microparticles (24). Thus, any reduction in efferocytosis is anticipated to result in the accumulation of DAMP-releasing late apoptotic/secondary necrotic cells, which in turn induce a pro-inflammatory cytokine profile. In previous clinical studies of patients with breast cancer, peripheral blood levels of the immunogenic cell death marker HMGB1 were increased within 3 days of the administration of the first chemotherapy cycle $(25,26)$. This result indicated that chemotherapy has an immediate effect on the elimination of dead cell remnants.

The present study investigated the efferocytotic capacity of the monocytes of patients with MM prior to and during bortezomib-based induction chemotherapy. Blood was taken prior to and during the first cycle of therapy and analyzed for efferocytosis, for the quantity of dead cell remnants, and for a panel of diverse cytokines, chemokines and soluble immune checkpoint molecules. The results revealed a clear impairment of efferocytosis in MM and give an overview of the immunological consequences.

\section{Materials and methods}

Patient population and sample collection. A total of 13 patients with MM ( 7 males and 6 females; age range $44-70$ years; mean age 62 years) and 12 healthy volunteers (6 males and 6 females; age range, 42-66 years; mean age, 59 years) were enrolled between November 2013 and September 2015 at the Vienna General Hospital (Vienna, Austria). Only patients without prior myeloma-specific therapy for at least 4 weeks were included. Patients receiving any other medications, ongoing or active HIV or hepatitis (B or C) infection were excluded from the study. All patients received a bortezomib-based regimen in various combinations with dexamethasone $(\mathrm{n}=8)$, doxorubicin $(n=6)$, cyclophosphamide $(n=4)$ or thalidomide $(n=3)$, in accordance with the international guidelines (27) and as described previously (28). Bone marrow aspirates (BMA) were collected at the time of diagnosis and following induction therapy. Mononuclear cells (MNCs) were prepared from the aspirates. Samples of peripheral blood were collected immediately prior to and 3, 7 and 10 days after the administration of the initial therapy cycle. These samples were used to prepare serum for chemokine and cytokine analysis, and PBMCs for cell analysis. Furthermore, patient monocytes from day 0 and day 3 were isolated from the peripheral blood samples and used for efferocytotic analysis. The study was approved by the Ethics Committee of the Medical University of Vienna (Vienna, Austria; ECS 1352/2013) and all participants provided written informed consent.

Cell preparation. MNCs and PBMCs were isolated by Ficoll centrifugation at $400 \mathrm{x}$ g for $20 \mathrm{~min}$ at room temperature from BMA or peripheral blood samples collected in EDTA-coated syringes or vacutainers. Cells were then washed in PBS and either used for direct analysis or for monocyte isolation using CD14 ${ }^{+}$microbeads (cat. no. 130-050-201; Miltenyi Biotec $\mathrm{GmbH}$, Bergisch Gladbach, Germany) according to the manufacturer's protocol. Briefly, PBMCs were incubated with $\mathrm{CD} 14^{+}$ microbeads for $15 \mathrm{~min}$ on ice. Following a washing step, the cell suspension was run through a LS column (Miltenyi Biotec $\mathrm{GmbH}$ ), which was installed on a MultiMACS Cell Separator (Miltenyi Biotec $\mathrm{GmbH}$ ). The flow-through was discarded. Next, the LS column was removed from the MultiMACS Cell Separator, flushed with buffer and the cell suspension containing $\mathrm{CD}_{14}{ }^{+}$monocytes was eluted into a fresh tube. Monocytes were used for the subsequent efferocytosis assay.

Cell analysis. Following isolation of PBMCs or MNCs, they were washed in PBS supplemented with 2\% bovine serum albumin (BSA; Merck KGaG, Darmstadt, Germany). For leukocyte typing, cells were then incubated with either anti-CD4-fluorescein isothiocyanate (FITC)/CD8-phycoerythrin (PE) (cat. no. 340039; BD Biosciences, Franklin Lakes, NJ, USA), anti CD14-FITC (cat. no. 17-0149-42; eBioscience; Thermo Fisher Scientific, Inc., Waltham, MA, USA) or anti CD138-FITC 
(cat. no. 11-1389-42; BD Biosciences) antibodies or the isotype control (cat. nos. 11-4752-80 and 17-4714-42, respectively; eBioscience; Thermo Fisher Scientific, Inc.) for 30 min on ice, following which the cells were washed. The antibodies were diluted according to the manufacturer's recommendations. A parallel sample was stained with 7-aminoactinomycin D (7-AAD; eBioscience; Thermo Fisher Scientific, Inc.) for $20 \mathrm{~min}$ at room temperature in the dark. For staining of dead cells, cells were washed in PBS at $45 \mathrm{~g}$ for $5 \mathrm{~min}$ at room temperature and then resuspended in $100 \mu 1 \mathrm{PBS}$ supplemented with $0.5 \mu 1$ Zombie Violet (BioLegend Inc., San Diego, CA, USA). For intracellular active caspase-3 staining, cells were fixed and permeabilized using an IntraPrep kit (Beckman Coulter, Inc., Brea, CA, USA) according to the manufacturer's protocol. Cells were then stained with anti-active caspase-3 (cat. no. 559565; BD Biosciences) antibody for 30 min on ice. Following another wash and a permeabilization step (IntraPrep Permeabilization Reagent; Beckman Coulter, Inc.), cells were incubated with an allophycocyanin (APC)-conjugated detection antibody (cat. no. 47-4714-82; eBioscience; Thermo Fisher Scientific, Inc.) for $30 \mathrm{~min}$ on ice. Subsequently, the cells were immediately analyzed with a Gallios flow cytometer (Beckman Coulter, Inc.,) using the Kaluza Analysis software (v.1.3; Beckman Coulter, Inc.).

Efferocytosis assay. Following isolation of patient monocytes, the efferocytosis assay was performed as described previously (23). Briefly, carboxyfluorescein succinimidyl ester (CFSE)-labeled (Invitrogen; Thermo Fisher Scientific, Inc.) Jurkat cells (ATCC-LGC Standards GmbH, Wesel, Germany) were cultured in Dulbecco's modified Eagle's Medium/Ham's F-12 including GlutaMAX(Invitrogen; Thermo Fisher Scientific, Inc.) supplemented with $10 \%$ fetal calf serum (Linaris $\mathrm{GmbH}$, Dossenheim, Germany) at $37^{\circ} \mathrm{C}$ and in a humidified $5 \% \mathrm{CO}_{2}$ atmosphere. Cells were treated with $100 \mathrm{nM}$ bortezomib (kindly provided by the pharmacy of the General Hospital of Vienna) for $24 \mathrm{~h}$ in serum-free Ultra Culture (UC) medium (Lonza Group, Ltd., Basel, Switzerland), washed to remove the drug and then incubated for $1 \mathrm{~h}$ in UC medium supplemented with $25 \%$ normal human serum (NHS; PAA Laboratories; GE Healthcare, Chicago, IL, USA). For co-culture, 100,000 apoptotic Jurkat cells were added to 100,000 monocytes and incubated for $2 \mathrm{~h}$ at $37^{\circ} \mathrm{C}$ in a total volume of $200 \mu \mathrm{l} \mathrm{UC}$ medium. Next, the cells were washed with PBS supplemented with $2 \%$ BSA and stained with an APC-conjugated anti-CD14 antibody (cat. no. 17-0149-42; eBioscience; Thermo Fisher Scientific, Inc.) for $30 \mathrm{~min}$ on ice. Cells were analyzed immediately using a Gallios flow cytometer. The efferocytotic index was calculated as follows: The $\mathrm{CFSE}^{+} / \mathrm{CD} 4^{+}$cell-population was divided by the total $\mathrm{CD} 14^{+}$cell count and multiplied by 100 .

Analysis of soluble immune modulators. Patient serum samples were collected and stored at $-80^{\circ} \mathrm{C}$ untilanalysis.IL-1 $\beta, \mathrm{IL}-6, \mathrm{IL}-8$, IL-10, IL-12 p70, IL-23, IFN- $\gamma$, TNF- $\alpha$, C-C motif chemokine ligand 2 (CCL2), chemokine (C-X-C motif) ligand 9 (CXCL9), CCL24, CXCL10, vascular endothelial growth factor-A (VEGF-A) and VEGF-C were quantified using a customized magnetic bead-based ProcartaPlex Multiplex Immunoassay (eBioscience; Thermo Fisher Scientific, Inc.). Soluble forms of the checkpoint molecules CD27, CD28, CD80/B7-1, CD137,
CD152/CTLA-4, CD223/LAG-3, herpes virus entry mediator (HVEM), B-and T-lymphocyte attenuator (BTLA), programmed death ligand 2 (PD-L2), PD-L1, programmed cell death protein 1 (PD-1), glucocorticoid-induced TNFR-related protein (GITR), indoleamine-pyrrole 2,3-dioxygenase (IDO) and T-cell immunoglobulin and mucin-domain containing-3 (TIM-3) were measured using a ProcartaPlex Human Immuno-Oncology Checkpoint Panel (cat. no. EPX140-15803-901; Affymetrix; Thermo Fisher Scientific, Inc.). All assays were performed according to the manufacturer's protocol and analyzed with a Luminex analyzer.

Statistical analysis. Multiple groups (Fig. 1A: Healthy volunteers, MM patients prior to therapy, and MM patients following therapy; Figs. 1B, 2B, 3A-F: Prior to therapy, 3, 7, and 10 days following therapy) were analyzed by one-way analysis of variance with the Greenhouse-Geisser correction. Comparisons between two groups (Fig. 1C and D: Prior to and following therapy, respectively) were assessed with an unpaired Student's $\mathrm{t}$-test. Data are expressed as the mean \pm the standard deviation. All statistical analyses were calculated using the GraphPad Prism software package version 6.04 (GraphPad Software, Inc., La Jolla, CA, USA).

\section{Results}

Efferocytosis by monocytes. Monocytes obtained from patients as well as healthy volunteers (HVs) exhibited an evidently higher uptake of apoptotic cell remnants than of viable prey cells (Fig. 1A). However, this difference was much lower with monocytes from patients with MM than with monocytes from HVs. The uptake of apoptotic cell remnants was similar prior to and 3 days after the administration of therapy. These data revealed that the efferocytotic capacity is markedly reduced in patients with MM compared with in healthy subjects. However, chemotherapy had no further effect on this monocytic cell function.

Dead cell remnants in blood and bone marrow. Whether the impaired efferocytosis of monocytes in patients with MM is associated with the accumulation of dead cells was then investigated. Considerable numbers of 7-AAD ${ }^{+}$PBMCs were observed prior to treatment (ranging between 0.6 and $6.7 \%$; Fig. 1B). This value did not change within 3 days of the administration of the first dose of therapy. But on day 7 all patients exhibited low numbers of $7-\mathrm{AAD}^{+} \mathrm{PBMCs}(<2.9 \%)$, remaining at this low level until day 10 . These data confirm that dead cells are present in the circulation of untreated patients with MM and indicate that the majority of these cells are cleared within the first 7 days of therapy. The $7 \mathrm{AAD}^{+}$dead cell remnants of untreated patients with MM could not be attributed to a specific immune cell subpopulation. They were negative for the three investigated clusters of differentiation markers (CD4, CD8 and CD14). These cell surface proteins may have been shed from the cell membrane during cell death.

The high number of dead cell remnants observed prior to chemotherapy is evidently due to the disease itself rather than to the chemotherapy. As MM leads primarily to destruction of the bone marrow, bone marrow was investigated in the next experiment. BMA were collected prior to and following 
A

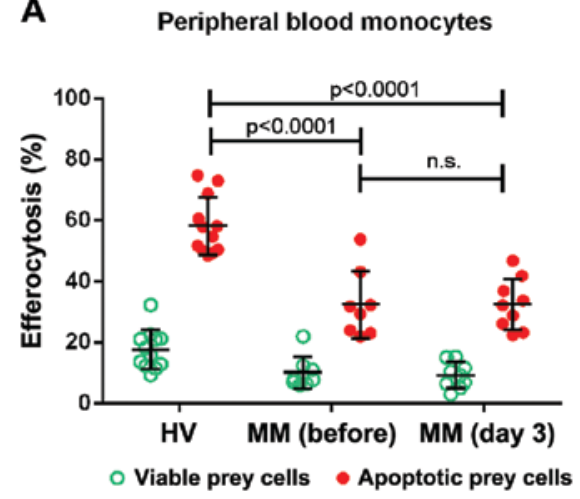

C

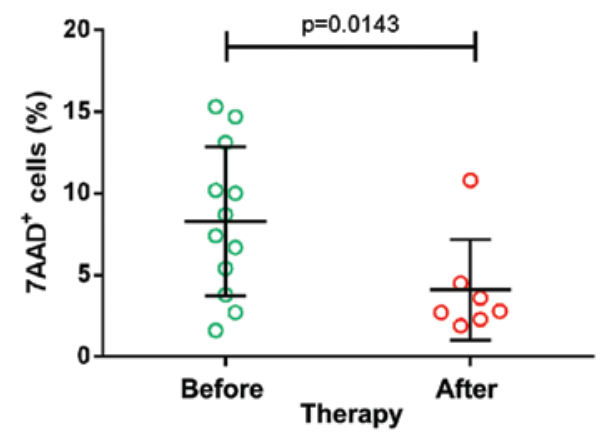

B

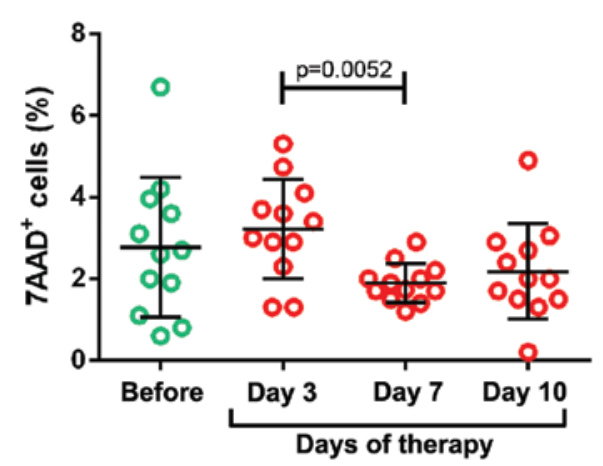

D

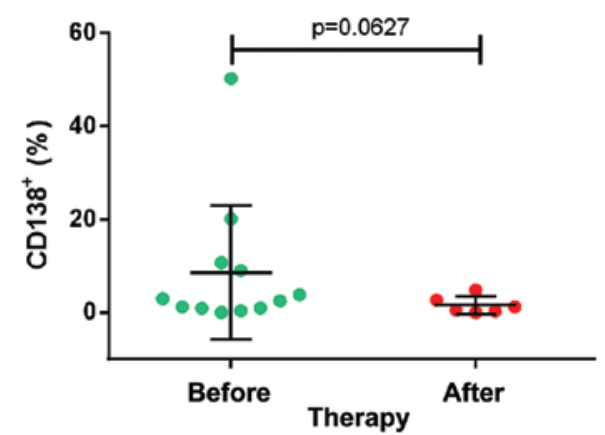

Figure 1. Efferocytosis and dead cell remnants. (A) Monocytes were isolated from HV and patients with MM prior to and after 3 days of induction therapy. Cells were co-cultivated with CFSE-labeled and either bortezomib-treated (apoptotic) or untreated (viable) Jurkat prey cells for 2 h. Next, the cell mixtures were stained with anti-CD14 antibody and analyzed by flow cytometry. The percentage of CFSE ${ }^{+}$cells within the CD14 ${ }^{+}$monocyte population was taken as the efferocytotic capacity. (B) PBMCs were isolated from patients with MM prior to and 3, 7, and 10 days after the administration of the first cycle of therapy. Cells were washed, stained with 7-AAD and analyzed by flow cytometry for the surface expression of CD4, CD8 or CD14. (C and D) Mononuclear cells from prior to and after therapy were isolated from bone marrow aspirates, washed and stained with (C) 7-AAD or (D) anti-CD138 antibody. The percentage of CD138 ${ }^{+}$and 7-AAD ${ }^{+}$cells is shown in the graphs. Each dot represents one patient at a given time point. The mean is depicted as a horizontal line. HV, healthy volunteer; CFSE, carboxyfluorescein succinimidyl ester; CD14, cluster of differentiation 14; PBMC, peripheral blood monocyte; 7-AAD, 7-aminoactinomycin D.

therapy, and the dead cells were stained with 7-AAD and analyzed by flow cytometry (a collection of bone marrow samples during therapy was not possible for ethical reasons). The analysis revealed the presence of considerable levels of $7-\mathrm{AAD}^{+}$cells prior to therapy (median, $8.05 \%$ of all cells in the aspirate; Fig. 1C). Notably, the level of 7-AAD ${ }^{+}$cells was strongly reduced following therapy. This reduction was associated with a reduction of the number of $\mathrm{CD} 138^{+}$plasma tumor cells (Fig. 1D).

The 7-AAD dye stains nucleated cells with a disintegrated cell membrane irrespective of whether the permeabilization of the cell membrane was a direct result of cell rupture (i.e. primary necrosis) or a secondary effect following apoptosis (i.e. late apoptosis/secondary necrosis). To estimate the contribution of secondary necrosis to 7-AAD ${ }^{+}$cells in patients with MM, cells were stained with antibodies against activated caspase 3 . The dead cell dye Zombie was used to identify cells with a disintegrated cell membrane. Fig. 2A describes the gating strategy, the results are shown in Fig. 2B. A mean of $18.4 \%$ of dead cells with a disintegrated cell membrane were positive for caspase 3 , indicating late apoptosis. The percentage of activated caspase $3^{+}$dead cells did not change during the observation period of the therapy. Taken together, these data indicate that untreated MM is associated with the presence a considerable number of dead cells in the bone marrow as well as in the blood flow, indicating a substantial number of late apoptotic/secondary necrotic cells.

Blood immune cells and serum levels of immunomodulatory molecules. To determine whether impaired efferocytosis and the accumulation of dead cells in patients with MM are associated with a pro-inflammatory response, the main PBMC subpopulations and the serum levels of a panel of immunomodulatory molecules were quantified. Fig. 3A demonstrates that the number of $\mathrm{CD} 14^{+}$monocytes remained unchanged over the course of chemotherapy, until day 7 when it increased slightly. By contrast, the number of $\mathrm{CD}^{+}$ regulatory and $\mathrm{CD}^{+}$cytotoxic $\mathrm{T}$ cells continuously declined (Fig. 3B and C). The panel of immunomodulatory molecules consisted of 14 different cytokines, chemokines and angiogenesis factors (TNF, IFN- $\alpha$, IL-1 $\beta$, IL-6, IL-8, IL-10, IL-12 p70, IL-23, CCL2, CCL24, CXCL9, CXCL10, VEGF-A and VEGF-C), as well as 14 soluble forms of co-stimulatory or co-inhibitory receptors (sPD-1, sPD-L1, sPD-L2, sTIM-3, sCTL-A4, sIDO, sCD27, sCD28, sCD80, sCD137, sHVEM, sLAG-3, sBTLA and sGITR). The results of all parameters are summarized in Table I. In total, 12 of these molecules were under the detection limit in all samples. The majority of the remaining molecules were present at stable levels throughout the therapy. Only sCD27, CCL2 and CCL24 were affected 


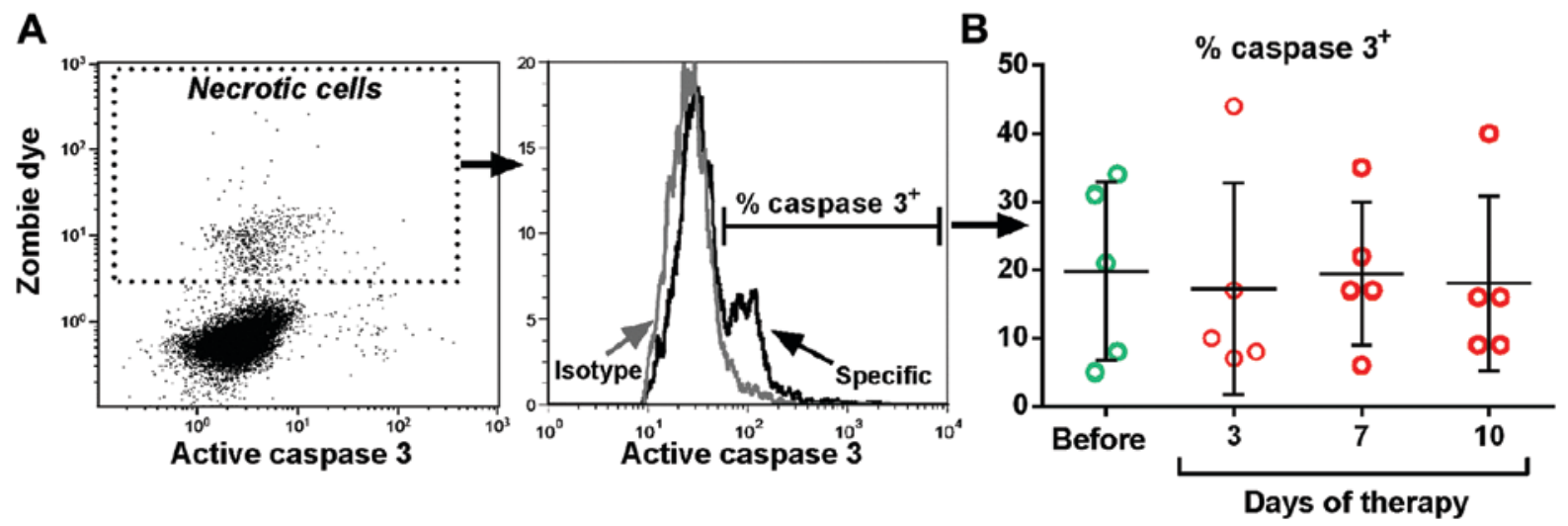

Figure 2. Contribution of apoptosis to circulating dead cell remnants. PBMCs were isolated from patients with MM prior to and 3, 7, and 10 days after the administration of the first cycle of therapy. Cells were stained for disintegrated cell membrane using the dead cell dye Zombie. Next, cells were permeabilized and stained for active caspase 3 using specific antibodies. (A) Flow cytometric analysis of the sample gating of the active caspase 3 signal against the Zombie signal. All cell events were included in the analysis. The box in the upper part of the graph indicates the dead cell remnants that were used to quantify the contribution of active caspase $3^{+}$cells to the total dead cell count. (B) Summary of the quantification. Each dot represents one patient at the indicated time point. The mean is depicted as horizontal line. PBMC, peripheral blood monocyte; MM, multiple myeloma.

A

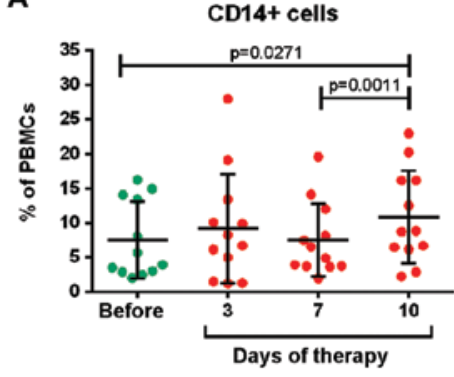

D

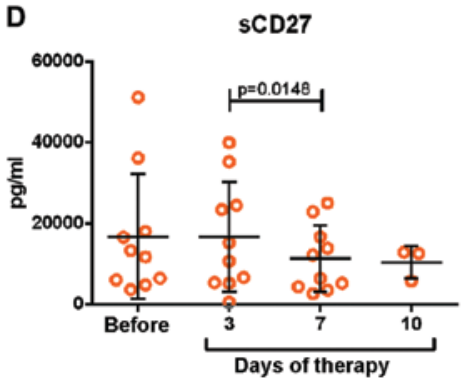

B

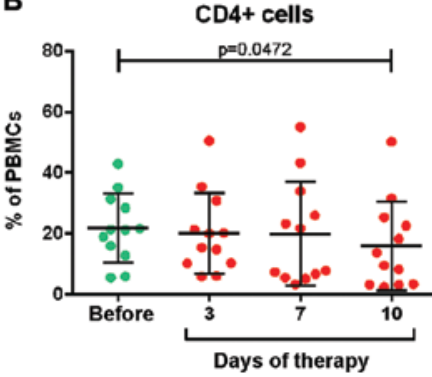

E

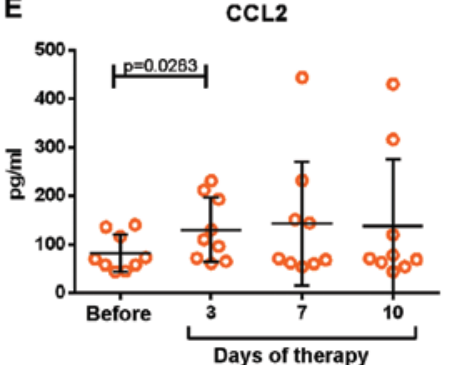

C

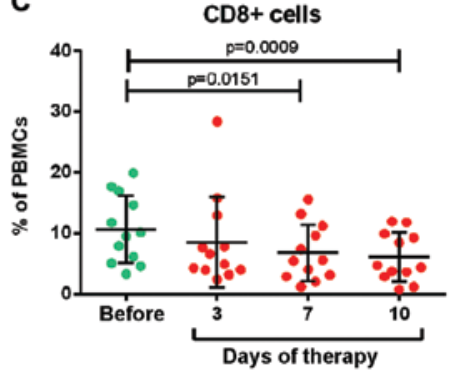

$\mathbf{F}$

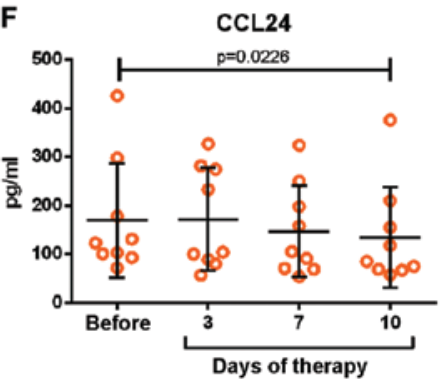

Figure 3. Effect of induction therapy on immune cells and immune-modulating soluble molecules. Blood was taken from patients with MM directly prior to and on day 3, 7, 10 after the administration of the first cycle of therapy. From all samples, serum and PBMCs were prepared. (A-C) Quantification of immune cells using antibodies against CD14, CD4 and CD8. The percentage of positive cells are shown. (D-F) Serum levels of sCD27, CCL2 and CCL24. Each dot represents one patient at the indicated time point. The mean is depicted as a horizontal line. Significant changes compared with the preceding time point or day 0 are indicated. PBMC, peripheral blood monocyte; MM, multiple myeloma; CD14, cluster of differentiation 14; CCL2, C-C motif chemokine ligand 2.

by bortezomib-based therapy (Fig. 3D-F). Prior to therapy, a strong variation in SCD27 was observed between patients. Notably, the high levels declined within seven days of therapy. By contrast, CCL2 levels strongly increased and those of CCL24 declined slightly. The results revealed no induction of classical pro-inflammatory cytokines or chemokines. Only a few immunomodulatory molecules were affected by the therapy.

\section{Discussion}

The present study revealed that monocytes in patients with MM have a reduced efferocytotic capacity. Impaired efferocytosis has also been observed in SLE (2), chronic granulomatous disease (9), Sjögren's syndrome (10), COPD (11), smokers (11), non-small cell lung cancer (29), non-eosinophilic asthma (12), and cystic fibrosis (30). To the best of our knowledge, this is the first report of diminished efferocytosis in MM. Notably, bortezomib-based induction had no negative effect on monocyte function or the monocyte count. However, a clear decline in the number of $\mathrm{CD}^{+}$and $\mathrm{CD}^{+} \mathrm{T}$-cells was observed. The in vivo data contradict those of previous in vitro studies, which stated that bortezomib specifically inhibited monocytes, leaving lymphocytes unaffected $(21,22)$. The reason for this difference is unclear; however, owing to large differences in the experimental 
Table I. Serum levels of immunomodulatory factors.

Concentration $^{\mathrm{a}}, \mathrm{pg} / \mathrm{ml}$

\begin{tabular}{|c|c|c|c|c|}
\hline Parameter & Day 0 & Day 3 & Day 7 & Day 10 \\
\hline TNF & n.d. & n.d. & n.d. & n.d. \\
\hline IFN- $\alpha$ & n.d. & n.d. & n.d. & n.d. \\
\hline IL-1 $\beta$ & n.d. & n.d. & n.d. & n.d. \\
\hline IL-6 & n.d. & n.d. & n.d. & n.d. \\
\hline IL-8 & n.d. & n.d. & n.d. & n.d. \\
\hline IL-10 & n.d. & n.d. & n.d. & n.d. \\
\hline IL-12 p70 & n.d. & n.d. & n.d. & n.d. \\
\hline IL-23 & n.d. & n.d. & n.d. & n.d. \\
\hline CCL2 & $82 \pm 44$ & $130 \pm 74$ & $143 \pm 128$ & $138 \pm 136$ \\
\hline CCL24 & $169 \pm 123$ & $172 \pm 113$ & $147 \pm 99$ & $135 \pm 105$ \\
\hline CXCL9 & $117 \pm 149$ & $154 \pm 196$ & $198 \pm 284$ & $176 \pm 275$ \\
\hline CXCL10 & n.d. & n.d. & n.d. & n.d. \\
\hline VEGF-A & $677 \pm 511$ & $687 \pm 567$ & $502 \pm 366$ & $680 \pm 554$ \\
\hline VEGF-C & $153 \pm 100$ & $179 \pm 94$ & $167 \pm 110$ & $134 \pm 73$ \\
\hline sPD-1 & $153 \pm 152$ & $136 \pm 151$ & $81 \pm 109$ & $198 \pm 109$ \\
\hline sPD-L1 & n.d. & n.d. & n.d. & n.d. \\
\hline sPD-L2 & $32,345 \pm 15,105$ & $32,731 \pm 17,049$ & $33,774 \pm 14,787$ & $47,711 \pm 24,709$ \\
\hline sTIM-3 & $15,963 \pm 5,024$ & $16,754 \pm 5,396$ & $15,847 \pm 4,797$ & $19,874 \pm 10,398$ \\
\hline sCTL-A4 & $112 \pm 150$ & $170 \pm 137$ & $172 \pm 144$ & $336 \pm 222$ \\
\hline sIDO & n.d. & n.d. & $423 \pm 1,043$ & $680 \pm 634$ \\
\hline sCD27 & $13,612 \pm 14,530$ & $14,305 \pm 13,459$ & $11,509 \pm 8,142$ & $10,419 \pm 6,136$ \\
\hline sCD28 & $132 \pm 178$ & $153 \pm 144$ & $159 \pm 143$ & $256 \pm 248$ \\
\hline sCD80 & $582 \pm 572$ & $594 \pm 570$ & $563 \pm 501$ & $1,322 \pm 844$ \\
\hline sCD137 & $206 \pm 273$ & $245 \pm 417$ & $135 \pm 188$ & $593 \pm 725$ \\
\hline sHVEM & n.d. & n.d. & n.d. & n.d. \\
\hline sLAG-3 & $295 \pm 292$ & $189 \pm 166$ & $234 \pm 273$ & $45 \pm 67$ \\
\hline sBTLA & $21,037 \pm 21,630$ & $25,758 \pm 28,507$ & $16,186 \pm 17,670$ & $29,916 \pm 24,860$ \\
\hline sGITR & n.d. & n.d. & n.d. & n.d. \\
\hline
\end{tabular}

${ }^{a}$ Mean \pm standard deviation. sTNF, soluble tumor necrosis factor; IFN- $\alpha$, interferon- $\alpha$; IL-1 $\beta$, interleukin- $1 \beta$; CCL2, C-C motif chemokine ligand 2; CXCL9, C-X-C motif chemokine ligand 9; VEGF-A, vascular endothelial growth factor-A; PD-1, programmed cell death protein 1; PD-L1, programmed death-ligand 1; TIM-3, T-cell immunoglobulin and mucin-domain containing-3; CTL-A4, cytotoxic T-lymphocyte associated protein 4; IDO, indoleamine 2,3-dioxygenase; CD27, cluster of differentiation 27; HVEM, herpesvirus entry mediator; LAG-3, lymphocyte-activation gene 3; BTLA, B- and T-lymphocyte attenuator; GITR, glucocorticoid-induced TNFR family related protein; n.d., not detectable.

setting, comparisons between in vitro and in vivo data should be conducted with care. Corroboration for the results of the present study comes from a report by Blanco et al (31), which demonstrated that bortezomib induced selective depletion of alloreactive T lymphocytes (31); however, the authors did not include monocytes in their analysis.

The reduced efferocytosis in patients with untreated MM was accompanied by an accumulation of dead cells. Approximately $20 \%$ of patients were positive for active caspase 3 , indicating that at least a proportion of the circulating dead cells were derived from late apoptotic/secondary necrotic cells. The disease-associated tissue damage in MM occurs mainly in the bone marrow, and an accordingly increased number of dead cell remnants were observed in this compartment. A previous report demonstrated an accumulation of apoptotic Annexin $\mathrm{V}^{+} / \mathrm{CD} 138^{+}$plasma cells in the bone marrow of patients with untreated MM (32). The authors revealed that a higher number of apoptotic plasma cells in the bone marrow was associated with an unfavorable prognosis (32). However, it is unclear whether the dead cell remnants in the circulation were derived from the bone marrow or if they were the consequence of a disease-associated direct effect on blood cells. The impaired efferocytotic capacity of circulating monocytes may contribute to the accumulation of dead cell remnants in the circulation, although the present study did not identify whether this is the only reason. The total number of $7-\mathrm{AAD}^{+}$dead cell remnants diminished during the first week of therapy. As no significant therapeutic effect on efferocytosis by monocytes could be observed, it was assumed that the lower number of $7-\mathrm{AAD}^{+}$cells results 
from the reduced formation of new dead cells in response to therapy. Thus, the lower degree of efferocytosis in patients with MM is evidently sufficient to clear the remaining dead cell remnants.

Leaky dead cell remnants release passive intracellular immunogenic DAMPs, which activate the Toll-like receptor signaling pathway in monocytes/macrophages, resulting in the secretion of pro-inflammatory mediators such as TNF, IL-1 $\beta$, IL-6, IL-8, IL-12p70, and IL-23 (33). No induction of any these cytokines was observed; however, an increase in CCL2 following administration of the first cycle of therapy was observed. Fraser et al (34) demonstrated that CCL2 is released from macrophages exposed to apoptotic cells in a Clq-dependent manner. Clq binds exclusively to a subpopulation of late apoptotic/secondary necrotic cells (26), indicating that the late apoptotic/secondary necrotic cells found in the present study may contribute to CCL2 formation. There is also a report claiming that CCL2 can be produced by tumor cells and tumor-associated fibroblasts (35). High CCL2 levels are associated with increased numbers of TAMs and poor patient prognosis in various types of cancer (35). Thus, the increased release of CCL2 may also result from the cytotoxic effects of the induction therapy on malignant plasma cells or stromal cells in the bone marrow. CCL2 is known to stimulate the emigration of inflammatory monocytes from the bone marrow into the circulating (35) to promote the survival of CD11b+ peripheral blood mononuclear cells (36) and to induce M2-type macrophage polarization (37). Thus, the increased CCL2 levels observed during induction therapy in MM may contribute to the sustained survival of monocytes during bortezomib treatment. Accordingly, a slight increase in the number of circulating monocytes was observed after 10 days of therapy. Notably, CCL2 levels increased during therapy in exactly those patients exhibiting high sCD27 levels prior to treatment, although no difference in CCL2 levels between $\mathrm{sCD} 27^{\text {high }}$ and $\mathrm{sCD} 27^{\text {low }}$ patients were observed prior to therapy, and the underlying reason remains unclear. The cell surface form of CD27 is highly expressed on normal plasma cells, T cells, B cells, NK cells and their precursors. Following cell activation, CD27 can be shed from the cell surface, thereby giving rise to circulating sCD27. Therefore, the soluble form is regarded as a marker for lymphoid cell activation (38). The shedding mechanism is mediated by the protein 'a disintegrin and metalloprotease', which is activated in response to extracellular ATP via P2X purinoreceptor 7 (39). As aforementioned, extracellular ATP is a well-known DAMP released by dead cell bodies with a disintegrating cell membrane. Thus, the concomitant reduction in dead cell remnants and sCD27 observed during the first cycle of therapy may be mutually associated. Notably, a prior study revealed that the in vitro treatment of human myeloma cells with bortezomib reduces the cell surface expression of CD27 (40). However, it is unclear whether this effect also occurs in vivo.

The present study revealed that MM is associated with reduced efferocytosis by monocytes, and with an increased number of dead cell remnants, including late apoptotic/secondary necrotic cells. However, the accumulation of late apoptotic cells was not associated with an inflammatory response. The bortezomib-based induction chemotherapy used to treat patients with MM inhibited neither the number nor the efferocytotic function of monocytes. However, it reduced the number of dead cell remnants in the circulation and the bone marrow. The origin and potential role of dead cell remnants in the pathophysiology of MM remains to be investigated in future studies.

\section{Acknowledgements}

The authors would like to thank Mr. Albert Müller and Dr Elisabeth Zechtl for their support in patient sample collection. This work was supported by the Austrian National Bank OeNB (grant no. 15661).

\section{References}

1. Poon IKH, Lucas CD, Rossi AG and Ravichandran KS: Apoptotic cell clearance: Basic biology and therapeutic potential. Nat Rev Immunol 14: 166-180, 2014

2. Birge RB, Boeltz S, Kumar S, Carlson J, Wanderley J, Calianese D, Barcinski M, Brekken RA, Huang X, Hutchins JT, et al: Phosphatidylserine is a global immunosuppressive signal in efferocytosis, infectious disease, and cancer. Cell Death Differ 23: 962-978, 2016.

3. Green DR, Oguin TH and Martinez J: The clearance of dying cells: Table for two. Cell Death Differ 23: 915-926, 2016.

4. Das A, Ganesh K, Khanna S, Sen CK and Roy S: Engulfment of apoptotic cells by macrophages: A role of microRNA-21 in the resolution of wound inflammation. J Immunol 192: 1120-1129, 2014.

5. Ferracini M, Rios FJ, Pecenin M and Jancar S: Clearance of apoptotic cells by macrophages induces regulatory phenotype and involves stimulation of CD36 and platelet-activating factor receptor. Mediators Inflamm 2013: 950273, 2013.

6. Stanford JC, Young C, Hicks D, Owens P, Williams A, Vaught DB Morrison MM, Lim J, Williams M, Brantley-Sieders DM, et al: Efferocytosis produces a prometastatic landscape during postpartum mammary gland involution. J Clin Invest 124: 4737-4752, 2014.

7. Murray PJ, Allen JE, Biswas SK, Fisher EA, Gilroy DW, Goerdt S, Gordon S, Hamilton JA, Ivashkiv LB, Lawrence T, et al: Macrophage activation and polarization: Nomenclature and experimental guidelines. Immunity 41: 14-20, 2014.

8. Silva MT: Secondary necrosis: The natural outcome of the complete apoptotic program. FEBS Lett 584: 4491-4499, 2010.

9. Fernandez-Boyanapalli RF, Falcone EL, Zerbe CS, Marciano BE, Frasch SC, Henson PM, Holland SM and Bratton DL: Impaired efferocytosis in human chronic granulomatous disease is reversed by pioglitazone treatment. J Allergy Clin Immunol 136: 1399-1401.e3, 2015.

10. Manoussakis MN, Fragoulis GE, Vakrakou AG and Moutsopoulos HM: Impaired clearance of early apoptotic cells mediated by inhibitory IgG antibodies in patients with primary Sjögren's syndrome. PLoS One 9: e112100, 2014.

11. Hamon R, Homan CC, Tran HB, Mukaro VR, Lester SE, Roscioli E, Bosco MD, Murgia CM, Ackland ML, Jersmann HP, et al: Zinc and zinc transporters in macrophages and their roles in efferocytosis in COPD. PLoS One 9: e110056, 2014.

12. Simpson JL, Gibson PG, Yang IA, Upham J, James A, Reynolds PN and Hodge S; AMAZES Study Research Group: Impaired macrophage phagocytosis in non-eosinophilic asthma. Clin Exp Allergy 43: 29-35, 2013.

13. Wickman G, Julian L and Olson MF: How apoptotic cells aid in the removal of their own cold dead bodies. Cell Death Differ 19: 735-742, 2012.

14. Aggarwal R, Ghobrial IM and Roodman GD: Chemokines in multiple myeloma. Exp Hematol 34: 1289-1295, 2006.

15. Caivano A, Laurenzana I, De Luca L, La Rocca F, Simeon V, Trino S, D'Auria F, Traficante A, Maietti M, Izzo T, et al: High serum levels of extracellular vesicles expressing malignancy-related markers are released in patients with various types of hematological neoplastic disorders. Tumor Biol 36: 9739-9752, 2015. 
16. Canella A, Harshman SW, Radomska HS, Freitas MA and Pichiorri F: The potential diagnostic power of extracellular vesicle analysis for multiple myeloma. Expert Rev Mol Diagn 16: 277-284, 2016

17. Ludwig H, Avet-Loiseau H, Bladé J, Boccadoro M, Cavenagh J, Cavo M, Davies F, de la Rubia J, Delimpasi S, Dimopoulos M, et al: European perspective on multiple myeloma treatment strategies: Update following recent congresses. Oncologist 17: 592-606, 2012.

18. Ludwig H, Beksac M, Bladé J, Boccadoro M, Cavenagh J, Cavo M, Dimopoulos M, Drach J, Einsele H, Facon T, et al: Current multiple myeloma treatment strategies with novel agents: A European perspective. Oncologist 15: 6-25, 2010.

19. Nooka AK, Kaufman JL, Behera M, Langston A, Waller EK, Flowers CR, Gleason C, Boise LH and Lonial S: Bortezomib-containing induction regimens in transplant-eligible myeloma patients: A meta-analysis of phase 3 randomized clinical trials. Cancer 119: 4119-4128, 2013.

20. Ribatti D, Nico B and Vacca A: Importance of the bone marrow microenvironment in inducing the angiogenic response in multiple myeloma. Oncogene 25: 4257-4266, 2006.

21. Straube C, Wehner R, Wendisch M, Bornhäuser M, Bachmann M, Rieber EP and Schmitz M: Bortezomib significantly impairs the immunostimulatory capacity of human myeloid blood dendritic cells. Leukemia 21: 1464-1471, 2007.

22. Arpinati M, Chirumbolo G, Nicolini B, Agostinelli C and Rondelli D: Selective apoptosis of monocytes and monocyte-derived DCs induced by bortezomib (Velcade). Bone Marrow Transplant 43: 253-259, 2009.

23. Liang YY, Arnold T, Michlmayr A, Rainprecht D, Perticevic B, Spittler A and Oehler R: Serum-dependent processing of late apoptotic cells for enhanced efferocytosis. Cell Death Dis 5 e1264, 2014

24. Liang YY, Rainprecht D, Eichmair E, Messner B and Oehler R: Serum-dependent processing of late apoptotic cells and their immunogenicity. Apoptosis 20: 1444-1456, 2015.

25. Arnold T, Michlmayr A, Baumann S, Burghuber C, Pluschnig U, Bartsch R, Steger G, Gnant M, Bergmann M, Bachleitner-Hofmann T and Oehler R: Plasma HMGB-1 after the initial dose of epirubicin/docetaxel in cancer. Eur J Clin Invest 43: 286-291, 2013.

26. Exner R, Sachet M, Arnold T, Zinn-Zinnenburg M, Michlmayr A, Dubsky P, Bartsch R, Steger G, Gnant M, Bergmann M, et al: Prognostic value of HMGB1 in early breast cancer patients under neoadjuvant chemotherapy. Cancer Med 5: 2350-2358, 2016.

27. Bird JM, Owen RG, D'Sa S, Snowden JA, Pratt G, Ashcroft J, Yong K, Cook G, Feyler S, Davies F, et al: Guidelines for the diagnosis and management of multiple myeloma 2011. $\mathrm{Br}$ Haematol 154: 32-75, 2011.
28. Lamm W, Wohlfarth P, Bojic M, Schörgenhofer C, Drach J, Gisslinger H, Worel N, Schiefer A, Schulenburg A, Agis H, et al: Outcome in multiple myeloma patients eligible for stem cell transplantation: A Single-center experience. Oncology 89: 196-204, 2015.

29. Dehle FC, Mukaro VR, Jurisevic C, Moffat D, Ahern J, Hodge G, Jersmann H, Reynolds PN and Hodge S: Defective lung macrophage function in lung cancer \pm chronic obstructive pulmonary disease (COPD/emphysema)-mediated by cancer cell production of PGE2? PLoS One 8: e61573, 2013.

30. McCubbrey AL and Curtis JL: Efferocytosis and lung disease. Chest 143: 1750-1757, 2013.

31. Blanco B, Pérez-Simón JA, Sánchez-Abarca LI, Carvajal-Vergara X, Mateos J, Vidriales B, López-Holgado N, Maiso P, Alberca M, Villarón E, et al: Bortezomib induces selective depletion of alloreactive $\mathrm{T}$ lymphocytes and decreases the production of Th1 cytokines. Blood 107: 3575-3583, 2006.

32. Minarik J, Scudla V, Ordeltova M, Bacovsky J, Pika T and Langova K: Prognostic significance of apoptotic index in multiple myeloma patients treated by conventional therapy and novel agents, thalidomide and bortezomib. Eur J Haematol 83: 528-534, 2009.

33. Krysko DV, Garg AD, Kaczmarek A, Krysko O, Agostinis P and Vandenabeele P: Immunogenic cell death and DAMPs in cancer therapy. Nat Rev Cancer 12: 860-875, 2012.

34. Fraser DA, Laust AK, Nelson EL and Tenner AJ: C1q differentially modulates phagocytosis and cytokine responses during ingestion of apoptotic cells by human monocytes, macrophages, and dendritic cells. J Immunol 183: 6175-6185, 2009.

35. Yadav A, Saini V and Arora S: MCP-1: Chemoattractant with a role beyond immunity: A review. Clin Chim Acta 411: 1570-1579, 2010.

36. Roca H, Varcos ZS, Sud S, Craig MJ and Pienta KJ: CCL2 and interleukin-6 promote survival of human CD11b+ peripheral blood mononuclear cells and induce M2-type macrophage polarization. J Biol Chem 284: 34342-34354, 2009.

37. Kobayashi M, Jeschke MG, Shigematsu K, Asai A, Yoshida S, Herndon DN and Suzuki F: M2b monocytes predominated in peripheral blood of severely burned patients. J Immunol 185: 7174-7179, 2010.

38. Lens SM, Tesselaar K, van Oers MH and van Lier RA: Control of lymphocyte function through CD27-CD70 interactions. Semin Immunol 10: 491-499, 1998.

39. Moon H, Na HY, Chong KH and Kim TJ: P2X7 receptor-dependent ATP-induced shedding of CD27 in mouse lymphocytes. Immunol Lett 102: 98-105, 2006.

40. Tagoug I, Plesa A and Dumontet C: Bortezomib influences the expression of malignant plasma cells membrane antigens. Eur J Pharmacol 706: 11-16, 2013. 